\title{
THE FIRST LOW-MASS BLACK HOLE X-RAY BINARY IDENTIFIED IN QUIESCENCE OUTSIDE OF A GLOBULAR CLUSTER
}

\author{
B. E. Tetarenko ${ }^{1}$, A. Bahramian ${ }^{1}$, R. M. Arnason ${ }^{1,2}$, J. C. A. Miller-Jones ${ }^{3}$, S. Repetto ${ }^{4,5}$, C. O. Heinke ${ }^{1}$, \\ T. J. Maccarone ${ }^{6}$, L. Chomiuk ${ }^{7}$, G. R. Sivakoff $^{1}$, J. Strader ${ }^{7}$, F. Kirsten ${ }^{3}$, and W. Vlemmings ${ }^{8}$ \\ ${ }^{1}$ Department of Physics, University of Alberta, CCIS 4-181, Edmonton, AB T6G 2E1, Canada; btetaren@ualberta.ca \\ ${ }^{2}$ Department of Physics and Astronomy, University of Western Ontario, 1151 Richmond Street, London, ON N6A 3K7, Canada \\ ${ }^{3}$ International Centre for Radio Astronomy Research (ICRAR), Curtin University, GPO Box U1987, Perth, WA 6845, Australia \\ ${ }^{4}$ Physics Department, Technion-Israel Institute of Technology, Haifa, 32000, Israel \\ ${ }^{5}$ Department of Astrophysics/IMAPP, Radboud University Nijmegen, PO Box 9010, NL-6500 GL Nijmegen, the Netherlands \\ ${ }^{6}$ Department of Physics, Texas Tech University, Box 41051, Lubbock, TX 79409-1051, USA \\ ${ }^{7}$ Department of Physics and Astronomy, Michigan State University, East Lansing, MI 48824, USA \\ ${ }^{8}$ Department of Earth and Space Sciences, Chalmers University of Technology, Onsala Space Observatory, SE-439 92 Onsala, Sweden \\ Received 2016 March 10; revised 2016 April 28; accepted 2016 May 1; published 2016 June 27
}

\begin{abstract}
The observed relation between the X-ray and radio properties of low-luminosity accreting black holes (BHs) has enabled the identification of multiple candidate black hole X-ray binaries (BHXBs) in globular clusters (GCs). Here, we report an identification of the radio source VLA J213002.08+120904 (aka M15 S2), recently reported in Kirsten et al., as a BHXB candidate. They showed that the parallax of this flat-spectrum variable radio source indicates a $2.2_{-0.3}^{+0.5} \mathrm{kpc}$ distance, which identifies it as lying in the foreground of the GC M15. We determine the radio characteristics of this source and place a deep limit on the X-ray luminosity of $\sim 4 \times 10^{29} \mathrm{erg} \mathrm{s}^{-1}$. Furthermore, we astrometrically identify a faint red stellar counterpart in archival Hubble images with colors consistent with a foreground star; at $2.2 \mathrm{kpc}$, its inferred mass is $0.1-0.2 M_{\odot}$. We rule out that this object is a pulsar, neutron star X-ray binary, cataclysmic variable, or planetary nebula, concluding that VLA J213002.08+120904 is the first accreting BHXB candidate discovered in quiescence outside of a GC. Given the relatively small area over which parallax studies of radio sources have been performed, this discovery suggests a much larger population of quiescent BHXBs in our Galaxy, $2.6 \times 10^{4}-1.7 \times 10^{8}$ BHXBs at $3 \sigma$ confidence, than has been previously estimated $\left(\sim 10^{2}-10^{4}\right)$ through population synthesis.
\end{abstract}

Key words: black hole physics - radio continuum: general - stars: individual (VLA J213002.08+120904) $\mathrm{X}$-rays: binaries

\section{INTRODUCTION}

Black hole X-ray binaries (BHXBs) are interacting binary systems where $\mathrm{X}$-rays are produced by material accreting from a secondary companion star onto a black hole $(\mathrm{BH})$ primary. Due to angular momentum in the system, accreted material does not flow directly onto the compact object; rather, it forms a differentially rotating disk around the BH known as an accretion disk (Shakura \& Sunyaev 1973). While some material accretes onto the $\mathrm{BH}$, a portion of this inward falling material may also be removed from the system via an outflow in the form of a relativistic plasma jet or an accretion disk wind (Blandford \& Konigl 1979; White \& Holt 1982). For major reviews of BHXBs see Chen et al. (1997), McClintock \& Remillard (2006), and Done et al. (2007).

Currently, the known Galactic BHXB population is made up of 19 dynamically confirmed BHs and 60 black hole candidates (BHCs), 56 of which are located in the field (Tetarenko et al. 2016b). The remaining four are found in globular clusters (GCs; Strader et al. 2012b; Chomiuk et al. 2013; Miller-Jones et al. 2015). The vast majority of these Galactic BHXBs are low-mass X-ray binaries (LMXBs) where mass transfer occurs via Roche lobe overflow of a secondary companion with a mass of $M_{2} \lesssim 3 M_{\odot}$ and spectral type A or later. In addition, most of these systems are transient, cycling between periods of quiescence and outburst. This behavior is associated with changing geometries of mass inflow and outflow (see, e.g., McClintock \& Remillard 2006).
While the majority of the known population has been discovered during outburst through bright $\mathrm{X}$-ray emission, typically peaking between $\sim 10^{36}$ and $10^{40} \mathrm{erg} \mathrm{s}^{-1}$ (e.g., Chen et al. 1997; Tetarenko et al. 2016b), the combination of more sensitive X-ray and radio telescopes has enabled the detection and identification of a handful of candidate quiescent $\mathrm{BH}$ LMXB systems in three Galactic GCs. Currently, no BH LMXBs known to exist in the field have been first identified outside of outburst and no X-ray outbursts have been clearly identified from candidate BH LMXBs in Galactic GCs. This is not surprising as it is far more difficult to identify quiescent BHs in the field, compared to those in GCs. Without established distances to field sources, simply having the ratio of X-ray to radio flux cannot strongly rule out background galaxies.

Typical estimates for the total number of BH LMXBs in the Galaxy have been computed both empirically using large-scale observational surveys (e.g., Romani 1998; Kalogera 1999; Corral-Santana et al. 2015), and theoretically from population synthesis codes (e.g., Romani 1992, 1994; Portegies Zwart et al. 1997; Kalogera \& Webbink 1998; Pfahl et al. 2003; Kiel \& Hurley 2006; Yungelson et al. 2006). These estimates span a wide range on the order of $\sim 10^{2}-10^{4}$. This large uncertainty is due to poorly constrained key characteristics that describe the Galactic binary population, including the $\mathrm{BH}$ natal kick distribution, the initial stellar mass function, the binary fraction, the distribution of binary periods and mass ratios, and the physics of common envelope and binary stellar evolution (e.g., Portegies Zwart \& Verbunt 1996; Belczynski et al. 2002; 
Pfahl et al. 2003; Ivanova et al. 2013). Detecting and identifying more of these systems is crucial to observationally constraining these uncertain parameters.

Accreting BHs are known to (i) emit compact radio jets in both the hard and quiescent spectral states (e.g., Gallo et al. 2004; Remillard \& McClintock 2006; see Done et al. 2007 for a description of X-ray spectral states) that produce spectrum ranging from flat to slightly inverted $(\alpha \sim$ 0.0-0.7, where $F_{\nu} \propto \nu^{\alpha}$; Blandford \& Konigl 1979; Fender et al. 2004a; Fender 2009; Russell et al. 2013a, 2013b), (ii) be more radio bright (i.e., have much higher radio luminosities at a given X-ray luminosity) than neutron stars (Fender \& Hendry 2000; Migliari \& Fender 2006), and (iii) exhibit a strong correlation between X-ray and radio emission in the hard and quiescent spectral states (e.g., Hannikainen et al. 1998; Corbel et al. 2000, 2003). As such, it is possible to distinguish between an accreting $\mathrm{BH}$ and an analogous neutron star or white dwarf system using a combination of X-ray and radio observations of a source. Specifically, we can use a technique, originally suggested by Maccarone \& Knigge (2007), that combines the established "universal" correlation between X-ray and radio luminosity in BHXBs (Gallo et al. 2003) with the spectral shape and position of a source in the radio/X-ray $\left(L_{\mathrm{R}} /\right.$ $\left.L_{\mathrm{X}}\right)$ plane.

Here, we report on the new BH candidate VLA J213002.08 +120904 (hereafter known as VLA J2130+12), which is the first field $\mathrm{BH}$ LMXB system identified by quiescent emission. VLA $\mathrm{J} 2130+12$ is located at a right ascension and declination of $21^{\mathrm{h}} 30^{\mathrm{m}} 02^{\mathrm{s}} .086$ and $12^{\circ} 09^{\prime} 04^{\prime \prime} 220$, respectively. Through a seven epoch VLBI campaign, Kirsten et al. (2014, hereafter K14) showed that VLA $\mathrm{J} 2130+12$, also known as M15 S2 (Knapp et al. 1996), is not associated with M15, and they argued that it is likely a field LMXB. K14 derive a parallax of $\pi=0.45 \pm 0.08$ mas, indicating a distance of $2.2_{-0.3}^{+0.5} \mathrm{kpc}$. Comparing this to M15's distance of $10.3 \pm$ $0.4 \mathrm{kpc}$ (van den Bosch et al. 2006) proves that VLA J2130 +12 is not related to M15. They find a proper motion for VLA $\mathrm{J} 2130+12$ of $\left(\mu_{\alpha}, \mu_{\delta}\right)=(-0.07 \pm 0.13,-1.26 \pm$ $0.29) \mathrm{mas} \mathrm{yr}^{-1}$, which is also inconsistent with the proper motion found by K14 for M15 members, average $\left(\mu_{\alpha}\right.$, $\left.\mu_{\delta}\right)=(0.58,4.05)$ mas $\mathrm{yr}^{-1}$. K14 found radio flux variations in VLA $\mathrm{J} 2130+12$ by a factor of 6 on timescales of a few months. Despite the known variability, they combined their $1.6 \mathrm{GHz}$ data with higher-frequency data reported in Knapp et al. $1996(230 \pm 40 \mu \mathrm{Jy}$ at $8.4 \mathrm{GHz})$ to suggest that the source has a flat radio spectrum. This would be indicative of an XRB.

In Section 2, we describe the observations and reduction of our Chandra and Very Large Array (VLA) data, as well as the archival Hubble Space Telescope (HST) data that we make use of. In Section 3, we present our observational results and argue for the presence of a stellar-mass BH in VLA $\mathrm{J} 2130+12$. In Section 4, we discuss the nature and system parameters of VLA $\mathrm{J} 2130+12$, refute alternative explanations, and provide an empirical estimate for the total number of BH LMXBs in the Galaxy. Finally, we provide a summary of our results in Section 5.

\section{OBSERVATIONS AND DATA REDUCTION}

\subsection{VLA Data}

In 2011 May, MAXI detected an X-ray flare in M15 (Morii et al. 2011). A Swift/XRT observation subsequently localized the event to the cluster core (Heinke et al. 2011), leading us to make Target-of-Opportunity observations with the Karl G. Janksy VLA, under program code 11A-269. Strader et al. (2012a) reported an analysis of the cluster core using these data, placing a limit on radio emission from a possible intermediate-mass $\mathrm{BH}$ at the cluster center. We observed M15 over five epochs for a total of $10 \mathrm{hr}$ (Table 1). The array was in its extended hybrid BnA-configuration on 2011 May 22, 26, and 30 , and in its most-extended A-configuration for the final two observations on 2011 August 21 and 22. We observed in two $1024 \mathrm{MHz}$ basebands, centered at frequencies of 5.0 and 7.45 GHz. Each baseband comprised eight spectral windows, each of which consisted of 64 spectral channels of width $2 \mathrm{MHz}$. We reduced the data using standard procedures within the Common Astronomy Software Application (CASA; McMullin et al. 2007). We used 3C 48 to set the amplitude scale (according to the Perley-Butler 2010 coefficients within the CASA task SETJY) and to perform both instrumental delay calibration and bandpass calibration. We used the nearby, compact calibrator $\mathrm{J} 2139+1423$ to determine the complex gain solutions that were interpolated onto the target field. The calibrated data were then imaged with natural weighting for maximum sensitivity. The initial calibration was good, and with only a few mJy of emission (dominated by a bright source at the edge of the field) we did not attempt self-calibration.

While the main science target was the outburst of M15 X-2 (Miller-Jones et al. 2011a), the source VLA J2130+12 was also within the field at the 94 and $87 \%$ response points of the primary beam at 5.0 and $7.45 \mathrm{GHz}$, respectively. We corrected the images for the effect of the primary beam response, and then measured the flux density of VLA J2130+12 (see Table 1) by fitting it with a point source model in the image plane, using the CASA task IMFIT. See Figure 1 for the radio image of VLA $\mathrm{J} 2130+12$.

In addition, we searched the NRAO archive for past data on M15 that can constrain the variability of M15 S2, and found a number of useful observations obtained with the historical (preupgrade) VLA, spanning 1989-2004 (see Table 2). All observations, with the exception of the 1990 April L-band observations, were obtained in the standard continuum mode of the historical VLA, with 2 intermediate frequency pairs, each of $50 \mathrm{MHz}$ bandwidth sampled in a single channel, and in full polarization. The 1990 April observations were obtained with 2 IFs, each of $50 \mathrm{MHz}$ bandwidth sampled with 7 channels (to minimize bandwidth smearing) and only one (right-hand) polarization. All data were edited and calibrated using standard routines in AIPS, and imaged using IMAGR in AIPS with a Briggs weighting scheme (robust $=1$ ). Flux densities were measured using JMFIT in AIPS.

\subsection{Chandra Data}

We analyzed all of the available non-grating Chandra/ACIS observations of M15 (Table 3). All of these observations were performed in faint mode and with the core of M15 on-axis. All of the observations were reduced and reprocessed using standard tools provided in CIAO 4.6 (Fruscione et al. 2006). After reduction, all of the observations were combined using the task reproject_obs to obtain a total exposure of $102 \mathrm{ks}$ (see Figure 2). Note that we also checked all of the other Chandra data (HRC and grating mode observations) for detections of VLA $\mathrm{J} 2130+12$, finding no evidence for increased activity. We performed all of our analyses in the $0.3-6.0 \mathrm{keV}$ band. 
Table 1

New 2011 VLA Observations of M15

\begin{tabular}{|c|c|c|c|c|c|c|}
\hline Date & MJD & $\begin{array}{l}\text { Duration } \\
\text { (minutes) }\end{array}$ & $\begin{array}{c}\text { Array } \\
\text { Configuration }\end{array}$ & $\begin{array}{c}5 \mathrm{GHz} \\
\text { Flux Density } \\
\left(\mu \mathrm{Jy} \mathrm{bm}^{-1}\right)\end{array}$ & $\begin{array}{c}7 \mathrm{GHz} \\
\text { Flux Density } \\
\left(\mu \mathrm{Jy} \mathrm{bm}^{-1}\right)\end{array}$ & $\begin{array}{l}\text { Spectral } \\
\text { Index }\end{array}$ \\
\hline 2011 May 22 & $55703.53 \pm 0.01$ & 60 & $\mathrm{BnA}$ & $508.1 \pm 9.1$ & $454.4 \pm 11.9$ & $-0.33 \pm 0.16$ \\
\hline 2011 May 26 & $55707.50 \pm 0.02$ & 60 & $\mathrm{BnA}$ & $393.1 \pm 10.0$ & $420.6 \pm 12.0$ & $0.17 \pm 0.11$ \\
\hline 2011 May 30 & $55711.51 \pm 0.06$ & 180 & $\mathrm{BnA}$ & $324.8 \pm 6.0$ & $337.9 \pm 8.5$ & $0.10 \pm 0.08$ \\
\hline 2011 Aug 21 & $55794.34 \pm 0.05$ & 150 & A & $302.0 \pm 12.5$ & $330.5 \pm 10.7$ & $0.23 \pm 0.15$ \\
\hline 2011 Aug 22 & $55795.44 \pm 0.05$ & 150 & A & $312.2 \pm 8.4$ & $334.4 \pm 7.9$ & $0.17 \pm 0.10$ \\
\hline
\end{tabular}

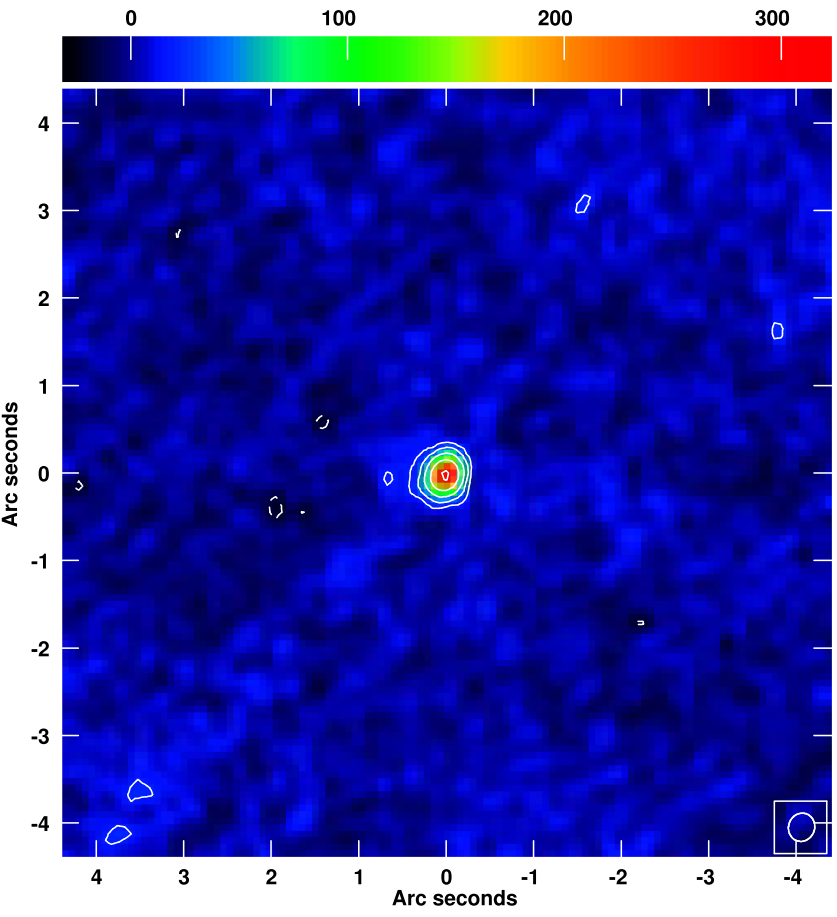

Figure 1. Radio image of VLA J2130+12 made from the data taken on 2011 August 21. The image was made with contour levels of $\pm 2^{n}$ times the lowest contour (with $n=1,2,3$ etc.), where the lowest contour level is three times the rms noise of $6.2 \mu \mathrm{Jy}$ beam $^{-1}$. The color bar is in units of $\mu \mathrm{Jy} \mathrm{beam}^{-1}$. The source position agrees exactly with the VLBI position of K14.

\subsection{HST Data}

We searched for the optical counterpart of VLA J2130+12 in data available from the Hubble Legacy Archive and Advanced Camera for Surveys (ACS) catalog (Sarajedini et al. 2007). The HST observations used in this work ${ }^{9}$ are tabulated in Table 4. We corrected astrometry (e.g., fitted for rotation, scale, and shifts) in HST images in two steps. First, we used the 2MASS catalog and corrected astrometry of ACS F814W and F606W images using matching coordinates of 40 relatively bright sources detected in both 2MASS and ACS images. We chose only those 2MASS sources whose ACS counterparts indicated that the 2MASS reported coordinates were not affected by blending of unresolved multiple bright sources. The 2MASS catalog absolute astrometry has a $1 \sigma$

\footnotetext{
9 Note that there are also WFPC2 observations of M15 in the F450W filter that covers the vicinity of VLA J2130+12. However, as WFPC2 has larger pixels, causing blending in crowded regions, we do not make use of these observations.
}

uncertainty of $\sim 0.12$ arcsec. $^{10}$ To increase the astrometric accuracy of the HST images further, in the second step, we used radio coordinates of two distinct sources detected in both the VLA radio and ACS images: AC 211 and M15 S1. These sources are clearly detected in the radio as reported by $\mathrm{K} 14$. AC 211 is clearly detected in all HST filters (identified through comparison with White \& Angelini 2001). K14 indicate that S1 is a background AGN. In ACS images, this source has a clear counterpart with a slightly extended point-spread function (PSF) consistent with a background galaxy. After this $(x, y)$ shift, our uncertainty in position in the HST images is reduced to $0.09 \operatorname{arcsec}(3 \sigma)$. We applied astrometry corrections to the WFC3 images by matching sources detected in ACS and WFC3 filters.

We find one star visible in the ACS/WFC images (F814W and F606W filters), 0."08 away from the radio position of VLA $\mathrm{J} 2130+12$ reported in $\mathrm{K} 14$. Based on values reported in the ACS catalog, the identified optical counterpart (See Figure 3) has an apparent (Vega) magnitude of $24.87 \pm 0.24$ in the $V$ band equivalent filter F606W and $23.09 \pm 0.12$ in $I(\mathrm{~F} 814 \mathrm{~W})$. In addition to the ACS survey images containing F814W and F606W filters, the vicinity of VLA J2130+12 was also observed with WFC3/UVIS in F438W, F336W, and F275W (Table 4). However, the counterpart is not clearly detected in single-epoch observations at any of these bands (See Figure 3).

\section{RESULTS}

\subsection{Radio Analysis}

Table 1 summarizes our fitted flux densities and derived spectral indices from each of our four epochs in 2011 May and August, and Table 2 summarizes the fitted flux densities for each available epoch in the archival VLA observations taken between 1989 and 2004. In the simultaneous 2011 data, the source is clearly variable (a factor of $\sim 1.5$ variation in our measured flux densities) and the spectral index is primarily slightly inverted, albeit with an excursion to steep when it is at its brightest in the first epoch (see Figure 4). In the archival data, we see a factor of $\sim 2$ variation in the measured flux densities at both 4.9 and $8.4 \mathrm{GHz}$.

Although the source is variable, we note that if we combine our 2011 VLA and archival VLA measurements with the flux density measurements reported in Knapp et al. (1996) of $230 \pm$ $40 \mu \mathrm{Jy}$ at $8.4 \mathrm{GHz}$, an estimated $5 \sigma$ upper limit of $150 \mu \mathrm{Jy}$ at $4.9 \mathrm{GHz}$ derived from archival data reported in Machin et al. (1990), and the flux density measurements at $1.6 \mathrm{GHz}$ reported

\footnotetext{
${ }^{10}$ See 2MASS All-Sky Data Release User guide, Section 2.f, http://www ipac.caltech.edu/2mass/releases/allsky/doc/sec2_2.html and Skrutskie et al. (2006).
} 
Table 2

Archival VLA Observations of M15

\begin{tabular}{|c|c|c|c|c|c|c|c|}
\hline Program ID & Date & MJD & $\begin{array}{l}\text { Duration } \\
\text { (minutes) }\end{array}$ & $\begin{array}{c}\text { Array } \\
\text { Configuration }\end{array}$ & $\begin{array}{c}1.49 \mathrm{GHz} \\
\text { Flux Density } \\
\left(\mu \mathrm{Jy} \mathrm{bm}^{-1}\right)\end{array}$ & $\begin{array}{c}4.86 \mathrm{GHz} \\
\text { Flux Density } \\
\left(\mu \mathrm{Jy} \mathrm{bm}^{-1}\right)\end{array}$ & $\begin{array}{c}8.44 \mathrm{GHz} \\
\text { Flux Density } \\
\left(\mu \mathrm{Jy} \mathrm{bm}^{-1}\right)\end{array}$ \\
\hline AM264 & 1989 Feb 19 & 47576.6 & 60 & $\mathrm{BnA}$ & $\cdots$ & $217.2 \pm 36.7$ & $\cdots$ \\
\hline $\mathrm{AJ} 186 / \mathrm{AK} 244^{\mathrm{a}}$ & 1990 Apr 09 & 47990.6 & 300 & A & $135.3 \pm 22.8$ & $\ldots$ & $\cdots$ \\
\hline AM308 & 1990 Jul 13 & 48085.3 & 96 & $\mathrm{BnA}$ & $\ldots$ & $\cdots$ & $210.6 \pm 18.7$ \\
\hline $\mathrm{AB} 0578^{\mathrm{b}}$ & $1991 \mathrm{Feb} 16$ & 48303.5 & 90 & $\mathrm{CnD}$ & $\ldots$ & $\ldots$ & $230 \pm 40$ \\
\hline $\mathrm{AJ} 211$ & 1991 Aug 08 & 48476.6 & 90 & A & $\ldots$ & $\ldots$ & $158.7 \pm 18.9$ \\
\hline AM342 & 1991 Dec 21 & 48611.9 & 42 & $\mathrm{BnA}$ & $\cdots$ & $345.1 \pm 35.0$ & $\ldots$ \\
\hline $\mathrm{AB} 1131$ & 2004 Oct 14 & 53292.9 & 324 & A & $\cdots$ & $\ldots$ & $221.9 \pm 8.4$ \\
\hline
\end{tabular}

Notes.

a AJ186 and AK244 data have been concatenated together, including observations on 1990 April 9, 10, 12, and 17 (47990.6, 47991.6, 47993.7, and 47998.6).

b Knapp et al. (1996).

Table 3

Chandra/ACIS Observations of M15

\begin{tabular}{llccc}
\hline \hline obsID & Instrument & Date & MJD & Exposure (ks) \\
\hline 0675 & ACIS & 2000 Aug 24 & 51780 & 19.8 \\
1903 & HRC-I & 2001 Jul 13 & 52103 & 9.1 \\
2412 & HRC-I & 2001 Aug 03 & 52124 & 8.82 \\
2413 & HRC-I & 2001 Aug 22 & 52143 & 10.79 \\
4572 & ACIS & 2004 Apr 17 & 53112 & 59.17 \\
9584 & HRC-I & 2007 Sep 05 & 54348 & 2.15 \\
11029 & ACIS & 2009 Aug 26 & 55069 & 34.18 \\
11886 & ACIS & 2009 Aug 28 & 55071 & 13.62 \\
11030 & ACIS & 2009 Sep 23 & 55097 & 49.22 \\
13420 & HRC-I & 2011 May 30 & 55711 & 1.45 \\
13710 & ACIS & 2012 Sep 18 & 56188 & 4.88 \\
14618 & HRC-S & 2013 Oct 07 & 56572 & 15.1 \\
\hline
\end{tabular}

in $\mathrm{K} 14(214 \pm 20 \mu \mathrm{Jy})$, we also find that VLA $\mathbf{J} 2130+12$ is consistent with flat-spectrum source. However, with this being said, the significant variability seen in the source could easily cause large errors on non-simultaneous spectral indices. As such, we caution against drawing any conclusions from spectral indices calculated with non-simultaneous data separated by years.

The variability of VLA $\mathrm{J} 2130+12$ that we observed is consistent with the factor of $\sim 6$ variation observed by K14 over the few-month timescale of their observations. However, since there was not enough flux in the field to be able to selfcalibrate, it is possible that uncorrected atmospheric phase wander (i.e., on timescales shorter than our phase calibration cycle time) may have led to some phase de-correlation, and hence introduced spurious variability to our measurements of VLA J2130+12. We therefore searched for background check sources in the field, whose measured amplitudes could be used to assess the likelihood of any phase de-correlation. While the previously known sources (AC211, M15 X-2, and source S1 of K14) all appeared to vary significantly, we found two sources located 2.5 arcmin to the south and west of the pointing center, with flux densities of 180 and $150 \mu \mathrm{Jy} \mathrm{bm}^{-1}$, respectively, that did not appear to vary significantly over the five epochs of observation. This gives us confidence that the observed variability of VLA $\mathrm{J} 2130+12$ (at a distance of 83 arcsec from the pointing center) is indeed real.

To determine the position of VLA J $2130+12$ on the $\left(L_{\mathrm{R}} / L_{\mathrm{X}}\right)$ plane, we derive a mean radio luminosity over all five 2011 observations. We find a luminosity of $(1.12 \pm 0.19) \times$
$10^{28} \mathrm{erg} \mathrm{s}^{-1}$ at $5 \mathrm{GHz}$ and $(1.61 \pm 0.26) \times 10^{28} \mathrm{erg} \mathrm{s}^{-1}$ at $7 \mathrm{GHz}$. The errors in luminosity are quoted to the $1 \sigma$ confidence level and include the uncertainties in flux density and distance. Note that here and throughout this paper, we calculate radio luminosity by assuming a flat radio spectrum up to the observed frequency (i.e., $L_{r}=4 \pi d^{2} \nu S_{\nu}$, where $S_{\nu}$ is the observed flux density, $\nu$ is the observing frequency, and $d$ is the distance to the source).

\subsection{X-Ray Analysis}

As the merged $102 \mathrm{ks}$ Chandra/ACIS image shows no evidence of an X-ray counterpart at the location of VLA J2130 +12 (see Figure 2), we estimate an upper limit on its brightness. Before estimating this upper limit, we first corrected the astrometry in the Chandra images. Due to heavy pile-up in the ACIS frames from AC211 and M15 X-2, it is difficult to obtain accurate localization of these sources to match radio coordinates. Thus, we corrected the absolute astrometry of the Chandra/ACIS image in two steps: (i) we corrected the astrometry of archival Chandra/HRC images using precise radio coordinates of AC211 (HRC detectors are not affected by pile-up), and (ii) we used coordinates of M15-X3 (Arnason et al. 2015) to match the ACIS image to HRC.

To estimate the upper limit we consider a $1^{\prime \prime}$ extraction radius, centered at the reported location of VLA $\mathrm{J} 2130+12$, which encircles $\sim 90 \%$ of the energy. ${ }^{11}$ M15 contains two persistent X-ray binaries in its core (AC 211 and M15 X2), both with $L_{\mathrm{X}} \geqslant 10^{36} \mathrm{erg} \mathrm{s}^{-1}$. These sources produce large PSF wings in Chandra observations. To consider the effect of these sources, we chose four background extraction regions around these two sources at the same angular distance as VLA J2130 +12 from them (see Figure 2). We estimate an upper limit on the count rate of $6.6 \times 10^{-5}$ counts $\mathrm{s}^{-1}$ (95\% confidence) in the $0.3-6 \mathrm{keV}$ band.

Using PIMMS, ${ }^{12}$ we convert this count rate to flux by assuming a photon index of 1.5, typical for LMXBs in the hard state (e.g., Campana et al. 1998, 2004), and a column density $N_{\mathrm{H}}=8.7 \times 10^{20} \mathrm{~cm}^{-2}$. This column density is calculated by assuming $R_{V}=3.1$, and using the reddening quoted for M15 (i.e., $E(B-V)=0.1)$ from the Harris Catalog, 2010 Edition (Harris 1996), in combination with the correlation between $A_{v}$

\footnotetext{
11 See Chandra Guide chap.4, Figure 4.23, at http://cxc.harvard.edu/ proposer/POG/html/chap4.html\#fg:hrma_ee_aspect_hrc_s_point_obs_ guide, for details.

${ }^{12}$ http://asc.harvard.edu/toolkit/pimms.jsp
} 


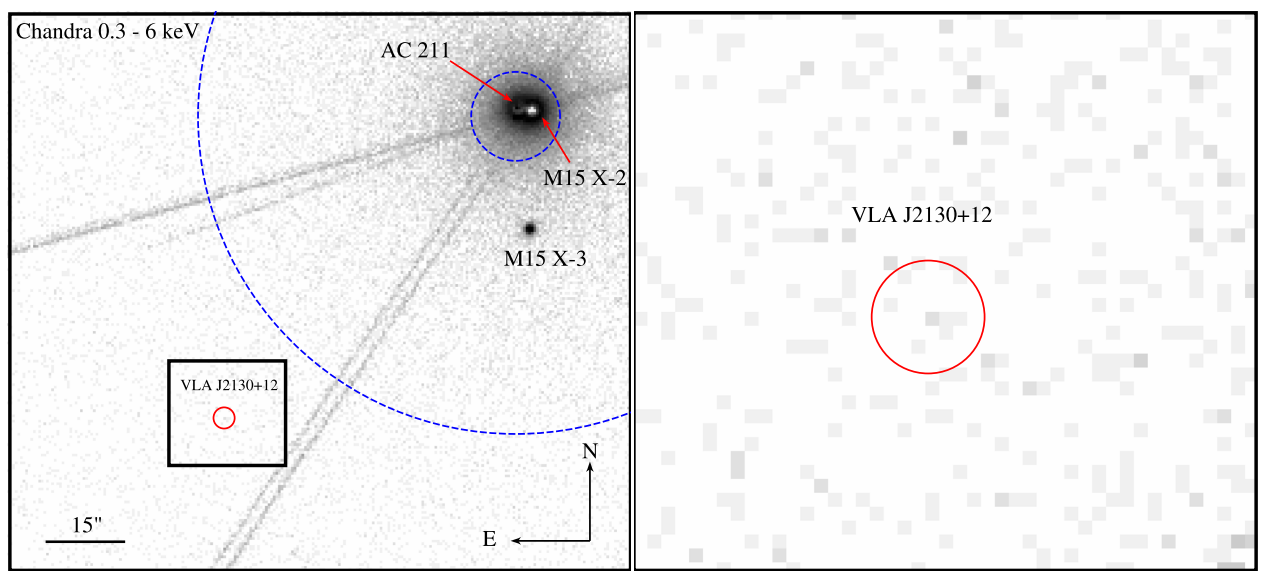

Figure 2. Left panel: the merged (102 ks) Chandra/ACIS image in the $0.3-6 \mathrm{keV}$ band. The red circle marks a 1" region around the location of S2 reported by K14. The locations of known sources in the field are also marked. AC211 and M15 X-2 are LMXBs (White \& Angelini 2001) and M15 X-3 is an X-ray transient (Heinke et al. 2009). The dashed blue contours mark the core and half-light radii of M15 (1 arcmin; Harris 1996). Right panel: a zoomed-in view of the black box region around VLA J2130+12 marked in the left panel. Note that the streaks in this image are artifacts caused by the large brightness of M15 X-2 and AC211.

Table 4

Archival HST Observations of M15 Used in this Work

\begin{tabular}{lcllr}
\hline \hline Proposal ID & Date & Detector & Band & Exposure (s) \\
\hline 10775 & 2006 May 02 & ACS/WFC & F814W & 615 \\
& & & F606W & 535 \\
12605 & \multirow{2}{*}{ 2011 Oct 16 } & WFC3/UVIS & F438W & 130 \\
& & & F336W & 700 \\
& \multirow{2}{*}{ 2011 Oct 22 } & \multirow{2}{*}{ WFC3/UVIS } & F275W & 1315 \\
& & F338W & 130 \\
& & & F275W & 1315 \\
\hline
\end{tabular}

and $N_{\mathrm{H}}$ from Foight et al. 2015 and Bahramian et al. 2015 (i.e., $N_{\mathrm{H}}=2.81 \times 10^{21} A_{v}$ ). As most of the reddening is occurring in the disk, this value is a valid approximation for VLA J2130 +12 , which is $2.2 \mathrm{kpc}$ away in the direction of M15. We find (95\% confidence) upper limits on flux in the $0.3-6 \mathrm{keV}$ and $1-10 \mathrm{keV}$ bands of $5.6 \times 10^{-16}$ and $6.4 \times 10^{-16} \mathrm{erg} \mathrm{cm}^{-2} \mathrm{~s}^{-1}$, respectively. Assuming a distance of $2.2 \mathrm{kpc}$, yields an upper limit on luminosity of $3.2 \times 10^{29} \mathrm{erg} \mathrm{s}^{-1}$ in the $0.3-6 \mathrm{keV}$ band and $3.7 \times 10^{29} \mathrm{erg} \mathrm{s}^{-1}$ in the $1-10 \mathrm{keV}$ band. With the derived radio and X-ray luminosities we have placed VLA J2130+12 on the $\left(L_{\mathrm{R}} / L_{\mathrm{X}}\right)$ plane (see Figure 5). Its position in the plane (in the radio-loud, $\mathrm{X}$-ray quiet regime) provides further evidence supporting the presence of a $\mathrm{BH}$ in the system.

We have also calculated (95\% confidence) upper limits on flux and luminosity in the $0.3-6 \mathrm{keV}$ band in all other available Chandra data (HRC and gratings; see Table 3) using the same method described above. In doing so, we find that in all Chandra observations the upper limits on the source have never been brighter than $4.6 \times 10^{-14} \mathrm{erg} \mathrm{cm}^{-2} \mathrm{~s}^{-1}$ (i.e., a few times $10^{31} \mathrm{erg} \mathrm{s}^{-1}$ at a distance of $2.2 \mathrm{kpc}$ ).

\subsection{Optical Analysis}

To determine whether or not the candidate counterpart identified in the ACS/WFC images is a cluster member, we plot the color-magnitude diagram of M15 using data from the ACS cluster survey (see Figure 6). Note that we have made use of the quality flag ${ }^{13}$ available in the ACS data sets on I- and

\footnotetext{
$\overline{13}$ The quality flag ranges from 0 (perfect quality) to 2.5 (bad quality).
}

V-band magnitudes for each source (Sarajedini et al. 2007), only plotting those points with quality flag values below 0.5 . The candidate optical counterpart is plotted in red. Note that the magnitudes of sources in the ACS catalog are based on multiple measurements and the errors plotted are the rms residuals of the individual measurements.

The candidate optical counterpart appears to be redder than the main sequence in M15. Compared to the distribution of main-sequence stars, this counterpart is a $3 \sigma$ outlier. While this strongly suggests that this object is not a member of the cluster, we note that there are other possibilities that could explain this observation (e.g., objects that appear redder than the main sequence due to unusual binary evolution paths, such as subsubgiants; Mathieu et al. 2003).

To determine how likely it is that the optical counterpart is in fact associated with VLA $\mathrm{J} 2130+12$ (rather then being a noncluster member in this direction of the sky), we determined the radius at which the next $3 \sigma$ outlier from the main sequence (plus horizontal branch) appears. This second outlier is 4."5 away from the radio coordinates; 237 sources, including the counterpart, are closer than this $3 \sigma$ outlier. Given that our likely optical counterpart is only 0 " 08 away and the second $3 \sigma$ outlier is $4 . .5$ from the radio coordinates of VLA J2130+12, we estimate the probability of finding a $3 \sigma$ outlier within a 0 ." 1 radius of these coordinates purely by chance to be $2 \times\left(0.1^{2}\right) /$ $\left(4.5^{2}\right)=0.1 \%$. Thus, we conclude that this star is likely to be the counterpart of VLA $\mathrm{J} 2130+12$.

To constrain the nature of the identified optical counterpart, we use Pysynphot to fit the $V$ and $I$ magnitudes. We use Allard et al's Phoenix models, with parameter inputs from Baraffe et al. (1998) and Baraffe \& Chabrier (1996) so we can associate $T_{\text {eff }} / \log g$ with a particular mass. Assuming that the source is at a distance of $2.2 \mathrm{kpc}$ (and is hence associated with the radio source), we fit two different models: the first, a main-sequence counterpart-only model, and the second, a two-component, main-sequence counterpart + power-law accretion disk model. Note that given that the upper limits from the other (WFC3) filters are not very constraining and it is likely that neither of the best-fit models would predict a significant detection in any of these bands, we only make use of the $V$ and $I$ magnitudes in this analysis. 

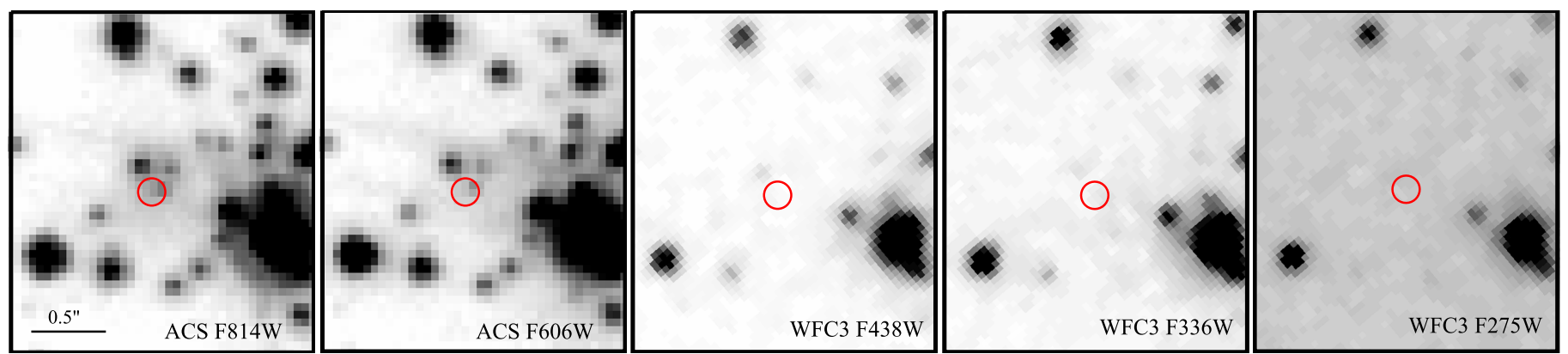

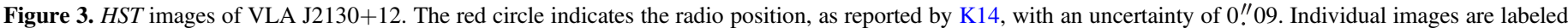

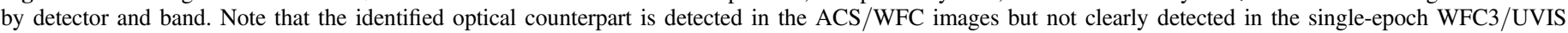
images (2011 October 22 data shown).

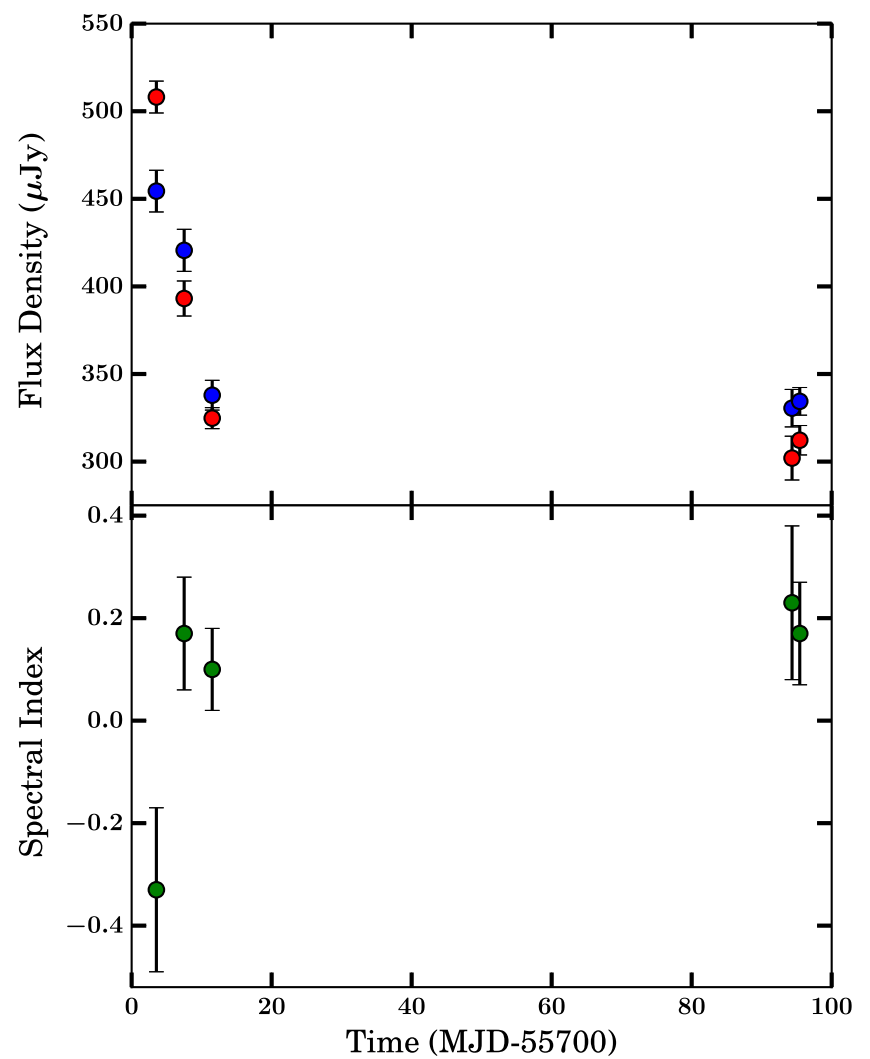

Figure 4. Radio frequency light curve of VLA J2130+12 (top) and corresponding spectral indices (bottom). In the top panel, red circles are the $5 \mathrm{GHz}$ measurements and blue circles are the $7 / 7.45 \mathrm{GHz}$ measurements.

The spectrum of an accretion disk that is purely viscously heated will have three regimes: a Rayleigh-Jeans regime at wavelengths longer than the Wien peak of the outermost annulus of the disk; a Wien spectrum at wavelengths shorter than the Wien peak of the innermost annulus of the disk; and a $\lambda^{-7 / 3}$ regime joining smoothly to the other two parts of the spectrum. As such, in this analysis, we fix the power-law index at $\Gamma_{\lambda}=-7 / 3$, corresponding to the "flat" part of an optically thick, geometrically thin accretion disk spectrum.

In both cases, we consider both a metal-poor ([M/ $\mathrm{H}]=-1.0)$ counterpart and a solar metallicity ([M/ $\mathrm{H}]=0.0)$ counterpart. The best-fit results are presented in Table 5. Overall, the fit results suggest a low-mass companion, between $\sim 0.1$ and $0.2 M_{\odot}$. The metal-poor star-only model produces an acceptable fit $\left(P_{\text {null }}>0.1\right)$, while the solar abundance star-only model produces a marginally unacceptable fit. Since the addition of a disk eliminates all degrees of freedom, we cannot calculate a null hypothesis probability. Instead, we can consider acceptable fits to occur when $\chi^{2}=0$. For comparison with $P_{\text {null }}>0.1$, we list all models within $\chi^{2}<2.71$, the $\chi^{2}$ that corresponds to $90 \%$ confidence interval for 1 degree of freedom. Similarly, we can compare the $\chi^{2}$ of the star and the star plus disk models. For the solar abundance model, the star plus disk model is consistent with no disk within the $95.7 \%$ confidence interval; for the metal-poor abundance model, the star plus disk model is consistent with no disk within the $75.6 \%$ confidence interval. Thus, the model fits are suggestive but not conclusive (i.e., not $3 \sigma$ ), so that we require an accretion disk to explain the counterpart's optical colors.

We also test the assertion that the identified counterpart is a member of M15 by fitting an additional model involving a main-sequence star at M15's distance $(10.3 \pm 0.4 \mathrm{kpc}$; van den Bosch et al. 2006) and metallicity. Here, we used the value quoted in the Harris catalog (Harris 1996) of $[\mathrm{Fe} / \mathrm{H}]=-2.37$ for the metallicity of M15. However, as Pysynphot's synthetic spectra use $[\mathrm{M} / \mathrm{H}]$ as inputs rather then $[\mathrm{Fe} / \mathrm{H}]$, we made the reasonable assumption (also used in Baraffe et al. 1998) that M15's $[\mathrm{Fe} / \mathrm{H}]$ translates to $[\mathrm{M} / \mathrm{H}] \approx-2.0$. As the $\chi^{2}$ values of this model are much poorer than those found for the star-only fits (assuming either solar metallicity or a metal-poor counterpart), the proposed optical counterpart is almost certainly not a cluster member (as argued previously; e.g., see green points in Figure 6), and is therefore likely to be associated with the radio source. In addition, since accretion disks are bluer than the main sequence, adding an accretion disk to a main-sequence model does not improve fits compared to the star-only model at the distance and abundance of M15.

Overall, given the previously discussed astrometric measurements, the significant radio variability observed on timescales of months, the observed flat to slightly inverted radio spectrum of the source, and the upper limit on the X-ray luminosity, it is likely that VLA J2130+12 is a foreground LMXB containing a $\mathrm{BH}$. If this is the case, then, to our knowledge, VLA J2130+12 is the most radio-loud quiescent $\mathrm{BH}$ source known to date.

While the radio to X-ray flux ratio for VLA J2130+12 is abnormally high, even for a quiescent BH (see Figure 5), we note that high radio/X-ray flux ratios have been observed to occur in near face-on systems (e.g., MAXI J1836-194; Russell et al. 2014). In addition, as some early works on advectiondominated accretion flows (Narayan \& Yi 1994) predict that the emission from the hot inner flow can start to fall out of the $\mathrm{X}$-ray band at very low Eddington fraction, it is possible that most of the emission in VLA $\mathrm{J} 2130+12$ is at far-UV rather 


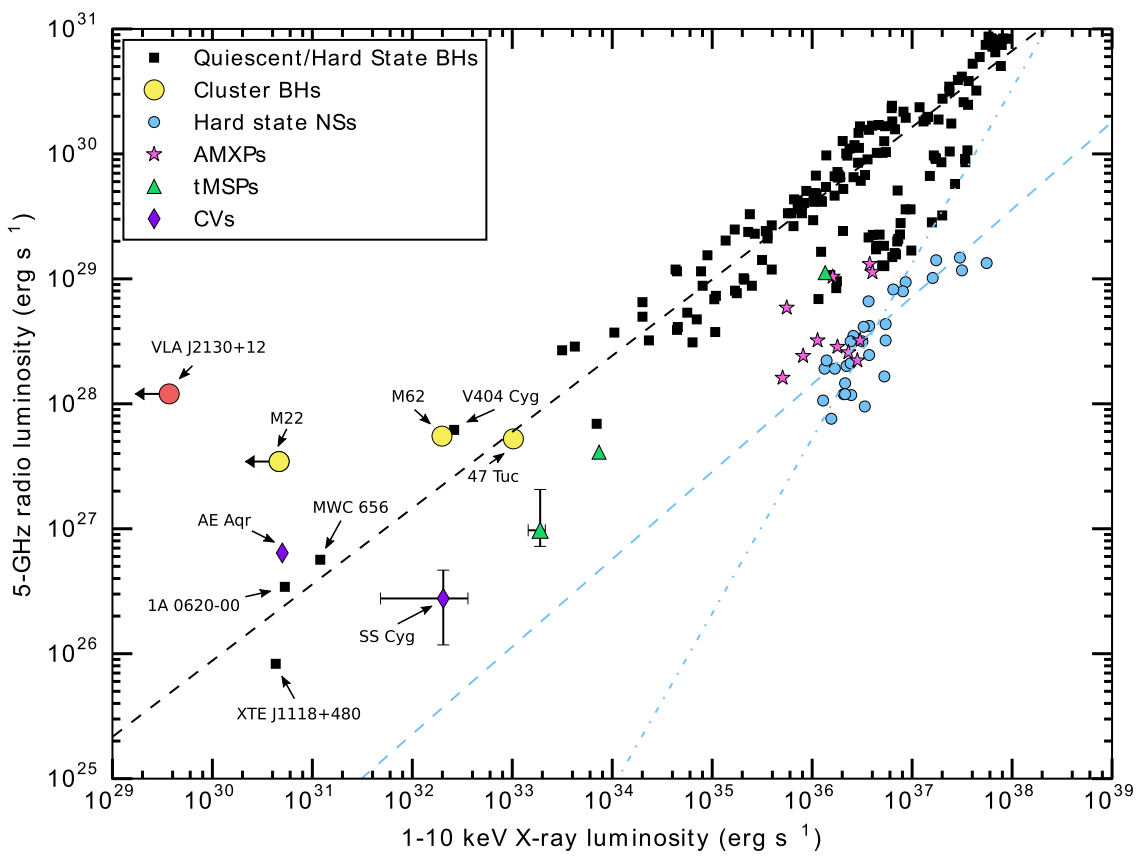

Figure 5. Radio/X-ray correlation for stellar-mass BHs in the hard and quiescent states. VLA J2130+12 is represented by the red circle. The black squares represent field BHs from the literature (Miller-Jones et al. 2011b; Gallo et al. 2012, 2014; Ratti et al. 2012; Corbel et al. 2013; Munar-Adrover et al. 2014; Dzib et al. 2015), and the yellow circles represent the BHCs in M22, M62, and 47 Tuc (Strader et al. 2012b; Chomiuk et al. 2013; Miller-Jones et al. 2015). The blue circles represent NS systems in the hard state (Rutledge et al. 1998; Moore et al. 2000; Migliari et al. 2003, 2010, 2011; Migliari \& Fender 2006; Tudose et al. 2009; Miller-Jones et al. 2010; Tetarenko et al. 2016a). Green triangles and pink stars show the binary transitional millisecond pulsar (Hill et al. 2011; Papitto et al. 2013; Deller et al. 2015) and accreting millisecond pulsar (Gaensler et al. 1999; Rupen et al. 2002; Fender et al. 2004b; Pooley 2004; Rupen et al. 2005; Migliari et al. 2011) systems, respectively. The purple diamonds show the CVs, AE Aqr (Eracleous et al. 1991; Abada-Simon et al. 1993) and SS Cyg (Russell et al. 2016), during bright flare/outburst periods in each source. These points are meant to represent the most radio-bright periods observed in these types of systems. The vast majority of CVs lie below these points in the $L_{\mathrm{R}} / L_{\mathrm{X}}$ plane (Fuerst et al. 1986; Kording et al. 2008, 2011; Byckling et al. 2010). The dotted black line shows the best-fit relation for BHs (Gallo et al. 2006), and the blue dashed and dashed-dotted lines show the two suggested correlations for NS systems (Migliari \& Fender 2006).

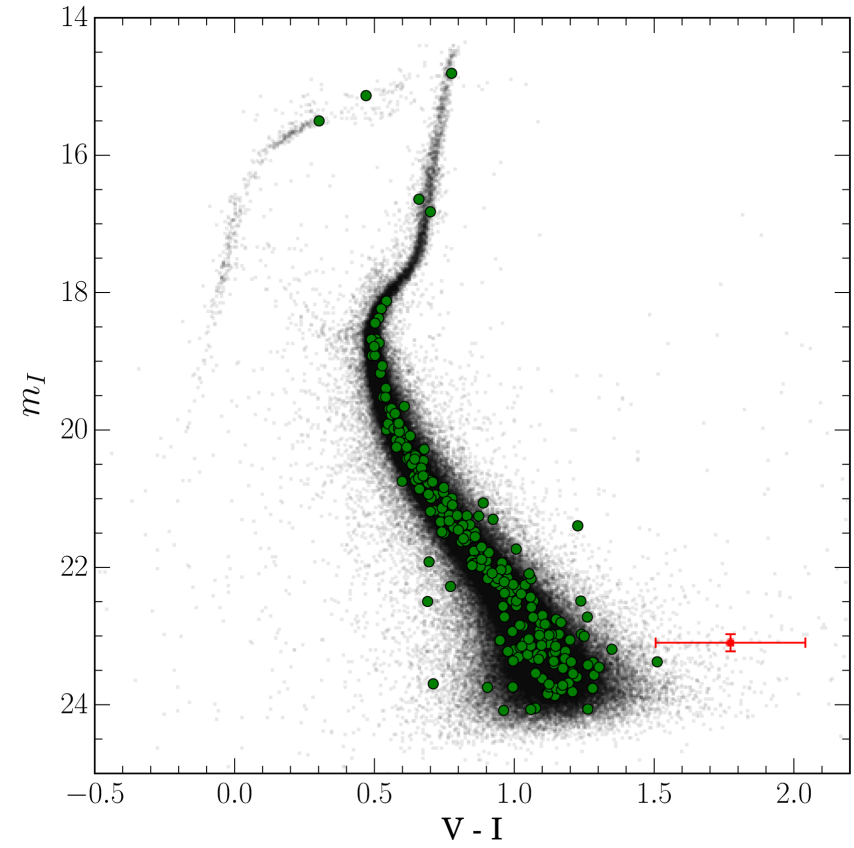

Figure 6. Color-magnitude diagram of M15 made using data from the ACS cluster survey. The data shown has been filtered by the quality flag for $I$ - and V-band magnitudes available in ACS data sets. Only points with quality flag values below 0.5 are plotted here. Green points indicate sources within a $4 .{ }^{\prime \prime} 5$ radius around the radio coordinates of VLA J2130+12. The identified optical counterpart of VLA J2130+12 is plotted in red. All uncertainties are directly quoted from the ACS database. The position of VLA J2130+12 strongly indicates that it is not a cluster member and therefore is most likely associated with the radio source (see text for details).
Table 5

Pysynphot Phoenix Model Fits

\begin{tabular}{lcclc}
\hline \hline Fit ID & & & \\
& $\begin{array}{c}\text { Metallicity } \\
\text { Assumption }\end{array}$ & $\begin{array}{c}\text { Counterpart Mass } \\
M_{\odot}\end{array}$ & $\chi^{2} /$ dof & $P_{\text {null }}$ \\
\hline star-only & solar & $0.150_{-0.010}^{+0.025}$ & $4.12 / 1$ & 0.04 \\
& metal-poor & $0.120 \pm 0.010$ & $1.36 / 1$ & 0.24 \\
& M15 & $0.330 \pm 0.010$ & $7.30 / 1$ & 0.007 \\
star+disk & solar $^{\mathrm{c}}$ & $0.145 \pm 0.010$ & $0.002 / 0$ & $\cdots$ \\
& & $0.185 \pm 0.010$ & $1.75 / 0$ & $\ldots$ \\
& metal-poor $^{\mathrm{d}}$ & $0.115 \pm 0.010$ & $0.006 / 0$ & $\ldots$ \\
& M15 $^{\mathrm{d}}$ & $0.330 \pm 0.010$ & $7.30 / 0$ & $\cdots$ \\
\hline
\end{tabular}

Notes. All errors are quoted at the $1 \sigma$ confidence level. For the solar and metalpoor fits, a distance of $2.2 \mathrm{kpc}$ is assumed. For the M15 metallicity fit, the distance to M15 $(10.4 \mathrm{kpc})$ is assumed.

${ }^{\text {a }}$ Star-only $=$ main-sequence counterpart only model, and star+disk $=$ mainsequence counterpart + power-law accretion disk model.

${ }^{\mathrm{b}}$ Metal-poor corresponds to $[\mathrm{M} / \mathrm{H}]=-1$, and M15 refers to the metallicity of the cluster of $[M / H] \approx-2$. See text for details.

${ }^{c}$ Note that we find two minima for this model. This can occur in the low-mass regime where one color $(V-I)$ can correspond to multiple magnitudes $\left(m_{v}\right)$. Given the data quality (i.e., zero dof), we cannot confidently prefer one fit over the other. In addition, the known distance to VLA J2130+12 also does not allow us to prefer one fit over the other.

${ }^{\mathrm{d}}$ Note that this fit returned a model with zero flux in the accretion disk.

${ }^{\mathrm{e}}$ Null hypothesis probability.

than X-ray frequencies, implying that the observed high radio/ $\mathrm{X}$-ray flux ratio could be explained in this case without invoking an enormous beaming factor. The large radio luminosity may also indicate a higher than average stellar- 
mass $\mathrm{BH}$ in the system if one applies the fundamental plane of BH activity (e.g., Merloni et al. 2003; Falcke et al. 2004). The implications of this possibility are discussed further in Section 4.2.

\section{DISCUSSION}

First, we discuss the nature of VLA J2130+12, offering alternative explanations that could possibly describe this source. Second, we discuss the binary properties of VLA $\mathbf{J} 2130+12$. Third, we discuss (i) how the discovery of this source is suggestive of a larger population of field BHXBs than has typically been assumed, and (ii) the implication that this larger number has for population synthesis modeling of these types of systems.

\subsection{The Nature of VLA J2130+12-Alternative Explanations}

While the X-ray, radio, and optical properties of VLA J2130 +12 indicate the presence of a stellar-mass $\mathrm{BH}$ (e.g., high radio/X-ray flux ratios, its specific position on the $L_{\mathrm{R}}-L_{\mathrm{X}}$ plane, photometry consistent with a main-sequence companion star plus potential accretion disk at the foreground distance of the radio source), here we discuss alternative explanations that could possibly produce the properties of this source. A variety of Galactic sources emit in the flux/luminosity range typical of $\mathrm{BHXBs}$ at X-ray and/or radio wavelengths. However, many of these objects can be ruled out using a combination of X-ray and radio flux ratios, the characteristic shape of the radio spectrum, and observations at other (optical/infrared) wavelengths (see, e.g., Seaquist 1993; Maccarone et al. 2012).

\subsubsection{Accreting Neutron Star Low-mass X-Ray Binaries (NS LMXBs)}

We can rule out NS LMXBs based on a combination of $\mathrm{X}$-ray/radio flux ratio and spectral index. Actively accreting NSs tend to be far less radio luminous (have much lower radio luminosities at a given X-ray luminosity) when compared to BH LMXBs (see, e.g., Migliari \& Fender 2006 and observe the measurements for NSs and transitional millisecond pulsars (tMSPs) in Figure 5). Moreover, as tMSPs enter pulsar mode when they are faint, at very low accretion rates for an NS, the tMSP scenario is not consistent with our observations. Therefore, we expect that it is exceptionally unlikely for an NS to be as radio luminous in quiescence as VLA $\mathrm{J} 2130+12$.

\subsubsection{Cataclysmic Variables (CVs)}

We can also rule out CVs based on their radio and X-ray fluxes. In Figure 5, we plot simultaneous radio/X-ray data for SS Cyg and AE Aqr during brief, bright flares/outbursts in each source. The vast majority of $\mathrm{CV}$ radio measurements are below the two points plotted (see, e.g., Fuerst et al. 1986; Kording et al. 2008, 2011; Byckling et al. 2010). While VLA $\mathrm{J} 2130+12$ is variable, at its faintest measured radio luminosity it is (i) nearly three orders of magnitude more luminous than the persistent quiescent emission detected from any known CV (e.g., Mason \& Gray 2007) and (ii) more than an order of magnitude more luminous than the brightest radio emission detected during the peak of flare/outburst events observed in nova-like CVs (e.g., Coppejans et al. 2015). As such, it is unlikely that a quiescent $\mathrm{CV}$ could reach radio luminosities as high as VLA $\mathrm{J} 2130+12$.

\subsubsection{Magnetars}

Magnetars can be radio bright, variable, have a flat spectrum, and show X-ray luminosities $<10^{31} \mathrm{erg} \mathrm{s}^{-1}$ (see Olausen \& Kaspi 2014), similar to VLA J2130+12. However, from both a theoretical and observational standpoint, magnetars are young objects; their observed positions with respect to the Galactic plane indicate an age of $<10^{4}$ years (Olausen \& Kaspi 2014). A lower limit on the age of VLA $\mathrm{J} 2130+12$ can be inferred by integrating the trajectory of the source backward in time from the observed Galactic position and proper motion, and computing the time at which the source crosses the Galactic plane (assumed as birthplace; see Section 4.2). To do so, we assume the distance to VLA $\mathrm{J} 2130+12$ to be uniformly distributed in the range $[1.9,2.7] \mathrm{kpc}$ (K14) and the proper motion to be distributed as a normal distribution in both the $\alpha$ and $\delta$ directions, taking as standard deviation the errors on the measurement. Since the line-of-sight velocity is not known, we model its value as being uniformly distributed in the range $[-300,300] \mathrm{km} \mathrm{s}^{-1}$. We integrate $5 \times 10^{4}$ orbits backward in time for 5 Gyr using the Python package for galactic dynamics, galpy (Bovy 2015), with a Galactic potential from Irrgang et al. 2013. By recording the times at which the binary crosses the Galactic plane, which we define as $z<0.1 \mathrm{kpc}$, we find a lower limit for the crossing time of $\approx 7 \mathrm{Myr}$. If, instead, we only integrate $5 \times 10^{4}$ orbits backward for the magnetar age limit of $10^{4}$ years, then we find that the source would have to have been born between 0.88 and $1.22 \mathrm{kpc}$ below the Galactic plane. Since this is a factor of four more distant from the Galactic plane than any known magnetar or magnetar candidate (Olausen \& Kaspi 2014), this clearly rules out the magnetar possibility.

\subsubsection{Planetary Nebula $(P N)$}

PNs emit optically thin thermal emission at radio wavelengths (and hence show a flat spectral index) and have low X-ray luminosities (i.e., $\sim 10^{30} \mathrm{erg} \mathrm{s}^{-1}$; Montez et al. 2010), both of which are consistent with VLA J2130+12. In fact, there is a known PN in M15 (K648; O'Dell et al. 1964) that is a $\sim 4 \mathrm{mJy} 5 \mathrm{GHz}$ radio source (Birkinshaw et al. 1981) and a weak X-ray source $\left(10^{31}-10^{32} \mathrm{erg} \mathrm{s}^{-1}\right.$, Hannikainen et al. 2005). However, no extended emission is observed around VLA $\mathrm{J} 2130+12$ in the wide array of archival HST images we have acquired. In addition, (i) the observed optical colors are not consistent with the presence of a hot white dwarf, which is expected to be within the PN itself, and (ii) the VLBI flux density measurements from $\mathrm{K} 14$ indicate brightness temperatures of $>4 \times 10^{6} \mathrm{~K}$, which are inconsistent with optically thin thermal emission $\left(<10^{4} \mathrm{~K}\right)$ from PNs. Finally, given that PNs typically lack any radio variability and display significantly extended radio emission (e.g., $3^{\prime \prime}$ for the more distant K648), neither of which apply to VLA J2130+12, this possibility is unlikely.

\subsubsection{Millisecond Pulsars (MSPs)}

While MSPs can display radio/X-ray flux ratios in the range of interest (see, e.g., Possenti et al. 2002; Maccarone et al. 2012), these sources typically display steep radio spectra that are inconsistent with the primarily flat spectrum we observe for VLA J2130+12. See, for example, Bates et al. (2013), who find a spectral index distribution with a mean of -1.4 and a standard deviation of 0.96 for these systems, or the rotation 
powered pulsars in the ATNF Pulsar Catalog (Manchester et al. 2005). Pulsars in the field can occasionally drive a wind that interacts with ambient material, called a pulsar wind nebula. However, while pulsar wind nebulae show a flat radio spectrum, they generally have X-ray luminosities of $L_{\mathrm{X}}>10^{34} \mathrm{erg} \mathrm{s}^{-1}$, large diameters, short lifetimes, and dense surroundings (Gaensler \& Slane 2006), all of which are inconsistent with our observations of VLA J2130+12. In addition, we note that the pulsar population in the field of M15 has been extremely well studied, ${ }^{14}$ including a thorough acceleration search by Anderson (1993). For VLA J2130+12 to be a millisecond radio pulsar, it would have to show several unusual features (a flat radio spectrum, a very small X-ray luminosity) and would have to be beamed away from Earth, which is unusual for millisecond pulsars considering their large beam sizes (e.g., Lyne \& Manchester 1988; Heinke et al. 2005).

\subsubsection{Ultracool Dwarfs}

While coronally active ultracool dwarfs (i.e., later than M6) can have radio/X-ray flux ratios in the range of interest and display flat radio spectra, these objects cannot be as radio luminous as VLA J2130+12( $L_{\mathrm{R}} \lesssim 10^{10}-10^{14} \mathrm{erg} \mathrm{s}^{-1}$; see, e.g., Berger et al. 2010; Maccarone et al. 2012). In addition, we note that these objects have only been observed to undergo moderate variability, and therefore it is likely that we are not just observing a flare in the source. As such, this possibility is ruled out.

\subsection{System Parameters of VLA J2130+12}

The binary counterpart masses of the Galactic BH LMXB population range from $\sim 0.18$ to $3.0 M_{\odot}$ (e.g., see Tetarenko et al. 2016b). With an estimated counterpart mass of $\sim 0.1-0.2 M_{\odot}$, VLA $\mathrm{J} 2130+12$ is one of the smallest known BHXB companions. Assuming a Roche lobe filling star (e.g., see Frank et al. 2002), we estimate an orbital period for the system of $\sim 1-2 \mathrm{hr}$, making VLA J2130+12 one of only seven known BH LMXBs that belong to the short-period $(<5 \mathrm{hr})$ regime (see Portegies Zwart et al. 2004; Tetarenko et al. 2016b).

The Galactic coordinates of VLA J2130+12 $(l, b)$ $=(65.009841,-27.334497)$, together with its distance, indicate a Galactic position at $(R, z) \approx(7.4,-1.0) \mathrm{kpc}$, where $R$ is the distance from the Galactic center projected onto the Galactic plane and $z$ is the distance from the Galactic plane. This $z$ places this system in the subset of BHXBs at large offset from the Galactic plane $(z \gtrsim 1 \mathrm{kpc}$; see Table 5 in Repetto et al. 2012, and Table 2 in Repetto \& Nelemans 2015). Given the set of orbital parameters and its current position, we can investigate whether or not the measured space velocity of VLA $\mathrm{J} 2130+12$ is consistent with an object originally in the disk that received a kick up into the halo.

Since BHXBs are thought to be formed in the plane of the Galaxy where most of the massive stars reside, a large distance from the plane is suggestive for a natal kick (NK) at birth, which is the velocity potentially acquired by a $\mathrm{BH}$ at formation. Assuming the binary received a velocity at birth perpendicular to the plane $V_{\perp}$ as a result of the $\mathrm{NK}$, and that it travels from its birthplace to the observed $z$, we can estimate a lower limit for

\footnotetext{
${ }^{14}$ See http://www.naic.edu/ pfreire/GCpsr.html for a list of the M15 discovered pulsars and relevant references.
}

its peculiar velocity at birth as $v_{\text {pec,min }}=V_{\perp}$. The $V_{\perp}$ is obtained by conservation of energy along the trajectory in the Galactic potential (see Repetto et al. 2012; Repetto \& Nelemans 2015). Accounting for the uncertainty in the distance to VLA $\mathrm{J} 2130+12$, we find a velocity $V_{\text {pec,min }}$ in the range $\approx 59-75 \mathrm{~km} \mathrm{~s}^{-1}$. To convert such velocity into an NK, we trace the binary evolution of the source backward in time, from the observed properties (BH mass, companion mass, and orbital period) until the $\mathrm{BH}$ formation (see, e.g., Repetto \& Nelemans 2015). Here we take a standard BH mass of $10 M_{\odot}$ and a companion mass of $0.2 M_{\odot}$, which give an orbital period of $P_{\text {orb }} \approx 2 \mathrm{hr}$. The orbital properties at $\mathrm{BH}$ formation, together with the lower limit on the peculiar velocity $v_{\text {pec,min }}$, deliver a minimum NK of $80-100 \mathrm{~km} \mathrm{~s}^{-1}$. Such a value for the NK would make this system similar to XTE J1118+480, a BHXB whose formation requires an $\mathrm{NK}$ of at least $\approx 80 \mathrm{~km} \mathrm{~s}^{-1}$ (Fragos et al. 2009). Hence, we conclude that VLA J2130+12 is consistent with having been formed in the disk and later kicked up into the halo of the Galaxy. This result potentially enlarges the sample of BHXBs that require an NK at formation.

There are four other known Galactic BH systems with a short period and high Galactic latitude like VLA J2130+12. It has been suggested that this subset of systems was also kicked out of the Galactic plane into the halo (e.g., see Kuulkers et al. 2013). We note that both this observation and our result are consistent with what is expected from theory, as wider orbits should be more easily disrupted when the $\mathrm{BH}$ forms and receives an NK due to the lower binding energy of the system. Meanwhile, tighter orbits can survive higher NKs (e.g., see Brandt \& Podsiadlowski 1995).

Given that one possible explanation for the anomalously high radio/X-ray flux ratio of VLA J2130+12 (compared to other quiescent $\mathrm{BHs}$ ) is that the system may contain a $\mathrm{BH}$ of $>10 M_{\odot}$, we have also considered what effect a larger mass would have on the computed NK. From a theoretical standpoint, the physics of the NK is not yet agreed upon (see, e.g., Fryer \& Kusenko 2006 for discussion). If, for example, the BH is formed via fallback onto the proto-neutron star and the NK is caused by asymmetric mass ejection in the supernova, then we would expect the NK to decrease with increasing BH mass (Fryer et al. 2012), as higher-mass BHs eject less mass at formation. However, (i) there are other equally viable models in which this is not the case (e.g., neutrino-driven NKs; Janka 2013; Belczynski et al. 2016) and (ii) observationally, there is no clear correlation seen between NK and BH mass (see Figure 12 in Repetto \& Nelemans 2015). Specifically, for our simulations, the only observational constraint we have is $V_{\text {pec,min }}$ (which has been computed assuming the binary was born in the Galactic plane, as described above). The peculiar velocity $V_{\text {pec }}$ that a binary acquires as a result of the $\mathrm{BH}$ formation is a combination of the $\mathrm{NK}$ and of the recoil that the binary receives as a consequence of the mass ejection in the supernova. The larger the total mass of the binary, the lower $V_{\text {pec }}$ is. Thus, a binary containing a larger-mass $\mathrm{BH}$ would require a larger NK to have the same peculiar velocity of a binary containing a lower-mass $\mathrm{BH}$. This implies that our calculated lower limit on the NK is a conservative estimate and would be valid even if the system did contain a $>10 M_{\odot} \mathrm{BH}$.

On the other hand, because the companion star is inferred to be of very low mass, we cannot exclude that the system was born longer than $10 \mathrm{Gyr}$ ago in the thick disk. The thick disk 
current scale height is $\approx 1 \mathrm{kpc}$ (Juric et al. 2008). Evolutionary sequences of BHXBs that host low-mass stars as companions $\left(M_{2} \approx 0.1-0.4 M_{\odot}\right)$ indicate a typical transferred mass to the $\mathrm{BH}$ in the range $0.7-1.5 M_{\odot}$ (see Table 3 in Fragos \& McClintock 2015). Hence, we cannot exclude that the companion star was $\approx 0.8 M_{\odot}$ on the zero-age main sequence, which would make a thick disk origin for VLA J2130+12 plausible. However, the scale height of the thick disk could have been much smaller in the past than it is currently (due to subsequent heating). Taking an initial distance from the Galactic plane of $z=0.3 \mathrm{kpc}$, the lower limit on the NK is only slightly affected: $74-92 \mathrm{~km} \mathrm{~s}^{-1}$. On the other hand, we also cannot exclude that the companion star was initially more massive than $\approx 1 M_{\odot}$, and thus we find the hypothesis of a birth in the thin disk to be the most likely explanation. Other possible origins, other than a birth in the Galactic plane, include (i) the formation and consequent ejection of the binary from a $\mathrm{GC}$, and (ii) the formation of the binary in the halo. However, since the metallicity of the companion star is currently not strongly constrained, it is not yet possible to assert the likelihood of these two scenarios.

Finally, with the determined set of orbital parameters we can theorize on the X-ray outburst properties of VLA J2130+12. Binary evolution indicates that short-period systems like VLA $\mathrm{J} 2130+12$ should not only exist but also should dominate the total BHXB number counts (e.g., Knevitt et al. 2014). Shortperiod XRB (BH and NS) systems are expected to have low peak outburst luminosities because their small disks only hold a small amount of mass (see King 2000; King \& Ritter 1998; Wu et al. 2010). In addition, it is thought that BH LMXBs are thought to be increasingly inefficient to X-ray luminosity with decreasing mass transfer rates (e.g., Knevitt et al. 2014), making outbursts in short-period BH LMXBs peak at even fainter luminosities. It has even been suggested that the temperature of the radiation during these outbursts could drop out of the X-ray band into the far-UV, so that even outbursts from nearby sources would not be detected by current surveys (Shahbaz et al. 1998; Wu et al. 2010; Maccarone \& Patruno 2013; Knevitt et al. 2014).

As such, given the estimated $P_{\text {orb }} \sim 1-2 \mathrm{hr}$, we would expect VLA $\mathbf{J} 2130+12$ (and other systems like it) to have peak $\mathrm{X}$-ray outburst luminosities that are well below (see Figure 2 in Knevitt et al. 2014) the typical detection threshold of the current all-sky and scanning instruments $\left(L_{\mathrm{bol}}<\mathrm{a}\right.$ few times $10^{36} \mathrm{erg} \mathrm{s}^{-1}$; Tetarenko et al. 2016b). Thus, it stands to reason that, as the all-sky and scanning instruments are our most effective resource for discovering new transients in the Galaxy, there is likely a huge population of extremely low accretion rate BHXBs within our Galaxy, yet to be discovered (e.g., see Maccarone et al. 2015).

\subsection{The Total Population of Field BH LMXBs in the Galaxy}

To estimate how many quiescent field BH LMXBs exist in the Galaxy, given that we find one such system in a random line of sight, we consider a simple population model for the density of BHXBs in the Galaxy. Given that VLA J2130+12 is located $\sim 1 \mathrm{kpc}$ below the plane (see Section 4.2 ) and the fact that the maximum detected distance from the plane of the Galaxy for BH LMXBs is $1.5 \mathrm{kpc}$ (Repetto et al. 2012), we start by modeling the Galaxy as a disk of radius $10 \mathrm{kpc}$ and thickness $3 \mathrm{kpc}$ ( $1.5 \mathrm{kpc}$ above and below the plane). This yields a total volume of $9.4 \times 10^{11} \mathrm{pc}^{3}$. Next, we uniformly distribute $N$ quiescent BHXBs like VLA J2130+12 within this volume. As BHs are more concentrated toward the plane than toward the halo (e.g., see Jonker \& Nelemans 2004; Repetto et al. 2012), this choice will give us a conservative lower limit on the number of BH LMXBs in the Galaxy.

Next, we determine the volume of space that has been searched for systems like VLA J2130+12. VLA J2130+12 was primarily identified as a candidate BHXB based on its parallax measurements and measured radio properties. All of the sources for the original project (K14) were originally chosen based on their properties measured in an Arecibo primary beam centered on the GC M15. Given that the Arecibo primary beam is $2^{\prime}$ in radius, the radius of the surveyed cone will be $1.3 \mathrm{pc}$ at $d=2.2 \mathrm{kpc}$. Considering the position of VLA $\mathrm{J} 2130+12$ at $b=-27^{\circ}$ and, furthermore, assuming it to be at the maximum distance of $1.5 \mathrm{kpc}$ below the plane would yield a maximum distance of $3.3 \mathrm{kpc}$ from Earth. Accordingly, this would yield a maximum radius of $1.9 \mathrm{pc}$ and in turn a total searched volume of $1.2 \times 10^{4} \mathrm{pc}^{3}$.

We note that the volume considered here should actually be the total volume of space wherein observed serendipitous radio sources have measured parallaxes. In other words, we need to account for the "number of trials" effect, whereby the predicted number of quiescent BH LMXBs would be scaled down by a factor equal to the number of observations like VLA J2130+12 that have been performed (e.g., wide field VLBI parallax). The field of view of VLBI observations is traditionally limited by both bandwidth and time-average smearing, effectively restricting the field of view to just a few arcseconds squared. As such, only a tiny portion of the primary beam of typical VLBI observations is actually analyzed. Additionally, as the space density of bright, highly compact radio sources is low, the entire field of view (even that limited by bandwidth/timeaveraged smearing) is not usually imaged because of the low probability of detecting sources other than the target source. We are not aware of similar searches for VLA J2130+12-like systems in other parallax observations, making the "number of trials" penalty very small, especially given the low flux density of VLA $\mathrm{J} 2130+12$.

Although this penalty is small, we can still provide a quantitative estimate based on a few assumptions. The observations of M15 that measured the parallax to VLA $\mathrm{J} 2130+12$ (K14) employed an interferometric technique where the position of a weaker potential in-beam calibrator source (M15 S1 and VLA J2130+12) can be measured against a brighter primary calibration source, with the potential for using the in-beam calibrator to transfer more accurate calibration solutions to other in-beam targets. Based on a literature review for papers where VLBI parallax measurements were determined using an in-beam calibrator, we find 24 similar measurements, the majority of which have been used to measure parallaxes to pulsars (Fomalont et al. 1999; Brisken et al. 2003; Chatterjee et al. 2005, 2009; Ng et al. 2007; Middelberg et al. 2011; Deller et al. 2012, 2013; Ransom et al. 2014; Liu et al. 2016). As these measurements represent additional opportunities to detect the parallax of a VLA J2130 +12 -like object, they could be considered as potential trials. However, all of the reported in-beam calibrators were significantly brighter (4-86 mJy) than VLA J2130+12 ( 0.1-0.5 mJy). Given our conservative assumption of a uniform volume density of VLA J2130+12-like objects in the Galaxy, we should down-weight the number of trials from 
brighter sources by $\left(f_{\nu \text {,in-beam }} / f_{\nu \text {, VLA } \mathbf{J} 2130+12}\right)^{-1.5}$. In that case, the number of trials penalty is only an additional $\sim 0.2$ trials. In addition, we note that the PSR $\pi$ parallax project (a large VLBA program) has reported ${ }^{15} 111$ additional in-beam calibrator sources that they used to measure parallaxes. Although they do not provide the flux densities of individual sources, they note that their median in-beam calibrator source is $9.2 \mathrm{mJy}$. We have measured the flux density function of secure 1-20 mJy FIRST sources (Helfand et al. 2015; $d N / d f_{\nu} \propto f_{\nu}^{-1.7}$ ) to estimate the expected distribution of the flux densities of in-beam calibrators. We found that the minimum flux density is likely $\sim 3.2 \mathrm{mJy}$, and using the same down-weighting we estimate an additional penalty of $\sim 0.9$ trials.

These estimates imply a total number of trials penalty of $\sim 2.1$ (including the VLA J2130+12 trial). We conservatively increase this penalty to 4 to account for any issues with our down-weighting scheme and unidentified analog observations. Considering this penalty, the fact that there are about 78 million volumes in the Galaxy similar to this one, and that the $3 \sigma$ confidence interval (Gehrels 1986) on the detection of 1 source is $0.0014-8.9$, we estimate $2.6 \times 10^{4}-1.7 \times 10^{8}$ objects (i.e., quiescently accreting BHXBs) like VLA J2130+12 in the Galaxy. The lower bound of this conservative estimate is consistent with the upper limit of the theoretical estimates for the number of $\mathrm{BH}$ LMXBs in the Galaxy computed from population synthesis codes $\left(\sim 10^{2}-10^{4}\right.$; Romani 1992, 1994; Portegies Zwart et al. 1997; Kalogera \& Webbink 1998; Pfahl et al. 2003; Kiel \& Hurley 2006; Yungelson et al. 2006). This implies that the population synthesis estimates for the numbers of BHXBs undergoing mass transfer in the Galaxy are likely underestimated.

We postulate that a likely reason for this discrepancy stems from the fact that these population synthesis estimates are generally calibrated from the bright XRB population, ignoring what is thought to be the most numerous class of XRBs, the socalled very faint X-ray transients, which are thought to be missing from the observed population because they undergo outbursts that are too faint to be detected by typical transient surveys.

\section{SUMMARY}

The known radio source VLA J2130+12 (Knapp et al. 1996) is not associated with the Galactic GC M15. Based on proper motion and parallax measurements, along with observed variability at radio wavelengths (on timescales of months), K14 suggested that VLA J2130+12 is a probable field LMXB. We argue that this system is likely to contain a stellar-mass $\mathrm{BH}$ accreting from a low-mass $\left(\sim 0.1-0.2 M_{\odot}\right)$ counterpart, making it the first known field BH LMXB candidate system identified in quiescence. This argument is supported by its X-ray and radio luminosities, radio spectra, and the identification of the likely optical counterpart.

First, we observe a flat to slightly inverted radio spectrum for the source, consistent with BHXBs in the quiescent and hard accretion states. In addition, we find a radio luminosity of $1.2 \times 10^{28} \mathrm{erg} \mathrm{s}^{-1}$ at $5 \mathrm{GHz}$ and an upper limit on X-ray luminosity of $3.7 \times 10^{29} \mathrm{erg} \mathrm{s}^{-1}$ in the $1-10 \mathrm{keV}$ band. These luminosities make VLA $\mathrm{J} 2130+12$ the most radio-loud quiescent $\mathrm{BH}$ candidate currently known, and puts VLA $\mathrm{J} 2130+12$ at a position in the $\left(L_{\mathrm{R}} / L_{\mathrm{X}}\right)$ plane that strongly rules

\footnotetext{
${ }^{15}$ https://safe.nrao.edu/vlba/psrpi/
}

out accreting neutron star and white dwarf systems. In addition, we note that all of the other alternate scenarios we have considered to describe VLA J2130+12 have been ruled out (see Section 4.1 for details).

Second, given the derived orbital parameters of VLA J2130 +12 (companion mass and orbital period), we find the current position of the system in the Galaxy to be consistent with it being born in the plane and receiving a large NK at birth ( $\gtrsim 80-100 \mathrm{~km} \mathrm{~s}^{-1}$ ), effectively launching it into the halo. We note that this finding adds VLA J2130+12 to the growing sample of (candidate and confirmed) BHXBs that require an NK at formation.

Third, we argue that the short orbital period ( $\sim 1-2 \mathrm{hr})$ of VLA $\mathrm{J} 2130+12$ would likely give rise to outbursts that radiate outside of the X-ray band, primarily in the UV. Making outbursts from VLA $\mathrm{J} 2130+12$, and other sources like it located within a few kpc of Earth, undetectable by current allsky and scanning X-ray instruments, which are largely our most effective resource for discovering new transients in the Galaxy to date.

Finally, using the fact that we observe one quiescent $\mathrm{BH}$ LMXB in a random line of sight, we estimate a total number of Galactic quiescent field BH LMXBs between $2.6 \times 10^{4}$ and $1.7 \times 10^{8}$, suggesting that a large population of extremely low accretion rate $\mathrm{BHXBs}$ exist within the Galaxy. Considering (i) the relatively small area over which deep radio/X-ray studies of radio-loud/X-ray quiet sources have been performed and (ii) the fact that the lower limit of this conservative estimate is consistent with the high end of the numbers computed from population synthesis codes, it becomes clear that to identify more of the Galactic BHXB population and further our understanding of the formation and evolution of these types of systems, large-scale radio or X-ray surveys are needed. Possible methods that could be used to accomplish this goal in the near-term include (i) taking multiple VLBA position measurements of bright, Galactic, flat-spectrum radio sources to look for high proper motions that would indicate nearby objects, accompanied by follow-up observations at other wavelengths upon detection, or (ii) performing repeated, deep, wide X-ray surveys of the bulge region (where most of the known $\mathrm{BH}$ LMXBs are located) to look for faint $\left(L_{X}\right.$ peak $<10^{36} \mathrm{erg} \mathrm{s}^{-1}$ ) X-ray outbursts of BH LMXBs that would normally go undetected by typical all-sky surveys, followed by observations at other wavelengths if one is detected. While these surveys should begin with current instruments, the next generation of both X-ray telescopes with large sensitivity over wide fields of view and radio arrays capable of more sensitive astrometry may be needed to identify and confirm the nature of large numbers of this population (e.g., Maccarone 2005; Fender et al. 2013).

The authors thank Tom Russell for providing the radio data for SS Cyg and Chris Done for useful discussions. B.E.T., G.R.S., and C.O.H. acknowledge support by NSERC Discovery Grants. J.S. acknowledges support from NSF grant AST-1308124. J.C.A.M.J. is the recipient of an Australian Research Council Future Fellowship (FT140101082). W.V. acknowledges financial support from the Swedish Research Council. The National Radio Astronomy Observatory is a facility of the National Science Foundation operated under cooperative agreement by Associated Universities, Inc. The search for an optical counterpart was based on observations 
made with the NASA/ESA Hubble Space Telescope, and obtained from the Hubble Legacy Archive, which is a collaboration between the Space Telescope Science Institute (STScI/NASA), the Space Telescope European Coordinating Facility (ST-ECF/ESA) and the Canadian Astronomy Data Center (CADC/NRC/CSA). The scientific results reported in this article are in part based on observations made by the Chandra X-ray Observatory. This research has made use of software provided by the Chandra X-ray Center (CXC) in the application package CIAO and the pysynphot package developed as part of STSDAS by the Space Telescope Science Institute (STScI). This publication makes use of data products from the Two Micron All Sky Survey, which is a joint project of the University of Massachusetts and the Infrared Processing and Analysis Center/California Institute of Technology, funded by the National Aeronautics and Space Administration and the National Science Foundation. This work has also made use of NASA's Astrophysics Data System (ADS).

\section{REFERENCES}

Abada-Simon, M., Lecacheux, A., Bastian, T., Bookbinder, J., \& Dulk, G. 1993, ApJ, 406, 692

Anderson, S. 1993, PhD thesis, California Institute of Technology

Arnason, R. M., Sivakoff, G. R., Heinke, C. O., Cohn, H. N., \& Lugger, P. M. 2015, ApJ, 807, 52

Bahramian, A., Heinke, C., Degenaar, N., et al. 2015, MNRAS, 452, 3475

Baraffe, I., \& Chabrier, G. 1996, ApJ, 461, 51

Baraffe, I., Chabrier, G., Allard, F., \& Hauschildt, P. H. 1998, A\&A, 337, 403

Bates, S. D., Lorimer, D. R., \& Verbiest, J. P. W. 2013, MNRAS, 431, 1352 Belczynski, K., Kalogera, V., \& Bulik, T. 2002, ApJ, 572, 407

Belczynski, K., Repetto, S., Holz, D., et al. 2016, ApJ, 819, 108

Berger, E., Basri, G., Fleming, T. A., et al. 2010, ApJ, 709, 332

Birkinshaw, M., Downes, A. J. B., \& Pooley, G. G. 1981, Obs, 101, 120

Blandford, R. D., \& Konigl, A. 1979, ApJ, 232, 34

Bovy, J. 2015, ApJS, 216, 29

Brandt, N., \& Podsiadlowski, P. 1995, MNRAS, 274, 461

Brisken, W., Thorsett, S. E., Golden, A., \& Goss, W. M. 2003, ApJ, 593, 89

Byckling, K., Mukai, K., Thorstensen, J. R., \& Osborne, J. P. 2010, MNRAS, 408, 2298

Campana, S., Israel, G. L., Stella, L., Gastaldello, F., \& Mereghetti, S. 2004, ApJ, 601, 474

Campana, S., Stella, L., Mereghetti, S., et al. 1998, ApJ, 499, 65

Chatterjee, S., Brisken, W. F., Vlemmings, W. H. T., et al. 2009, ApJ, 698, 250 Chatterjee, S., Vlemmings, W. H. T., Brisken, W. F., et al. 2005, ApJ, 630, 61

Chen, W., Shrader, C., \& Livio, M. 1997, ApJ, 491, 312

Chomiuk, L., Strader, J., Maccarone, T., et al. 2013, ApJ, 777, 69

Coppejans, D., Kording, E., Miller-Jones, J., et al. 2015, MNRAS, 451, 3801

Corbel, S., Coriat, M., Brocksopp, C., et al. 2013, MNRAS, 428, 2500

Corbel, S., Fender, R. P., Tzioumis, A. K., et al. 2000, A\&A, 359, 251

Corbel, S., Nowak, M., Fender, R. P., Tzioumis, A. K., \& Markoff, S. 2003, A\&A, 400, 1007

Corral-Santana, J., Casares, J., Munoz-Darias, T., et al. 2015, arXiv:1510. 08869

Deller, A. T., Archibald, A. M., Brisken, W. F., et al. 2012, ApJ, 756, 25

Deller, A. T., Boyles, J., Lorimer, D. R., et al. 2013, ApJ, 770, 145

Deller, A. T., Moldon, J., Miller-Jones, J. C. A., et al. 2015, ApJ, 809, 13

Done, C., Gierlinski, M., \& Kubota, A. 2007, A\&ARv, 15, 1

Dzib, S., Massi, M., \& Jaron, F. 2015, A\&A, 580, 6

Eracleous, M., Halpern, J., \& Patterson, J. 1991, ApJ, 382, 290

Falcke, H., Kording, E., \& Markoff, S. 2004, A\&A, 414, 895

Fender, R. 2009, in The Jet Paradigm-From Microquasars to Quasars, ed. T. Belloni (Berlin: Springer), 115

Fender, R., Belloni, T., \& Gallo, E. 2004a, MNRAS, 355, 1105

Fender, R., De Bruyn, G., Pooley, G., \& Stappers, B. 2004b, ATel, 361, 1

Fender, R. P., \& Hendry, M. A. 2000, MNRAS, 317, 1

Fender, R. P., Maccarone, T. J., \& Heywood, I. 2013, MNRAS, 430, 1538

Foight, D., Guver, T., Ozel, F., \& Slane, P. 2015, arXiv:1504.07274

Fomalont, E. B., Goss, W. M., Beasley, A. J., \& Chatterjee, S. 1999, AJ, 117,3025

Fragos, T., \& McClintock, J. 2015, ApJ, 800, 17
Fragos, T., Willems, B., Kalogera, V., et al. 2009, ApJ, 697, 1057

Frank, J., King, A., \& Raine, D. 2002, Accretion Power in Astrophysics (Cambridge: Cambridge Univ. Press)

Fruscione, A., McDowell, J., Allen, G., et al. 2006, Proc. SPIE, 6720, 1

Fryer, C., Belczynski, K., Wiktorowicz, G., et al. 2012, ApJ, 749, 91

Fryer, C. L., \& Kusenko, A. 2006, ApJ, 163, 335

Fuerst, E., Benz, A., Hirth, W., Kiplinger, A., \& Geffert, M. 1986, A\&A, 154,377

Gaensler, B., Stappers, B., \& Getts, T. 1999, ApJL, 522, L117

Gaensler, B. M., \& Slane, P. 2006, ARA\&A, 44, 17

Gallo, E., Fender, R., Maccarone, T., \& Jonker, P. 2004, PThPS, 155, 83

Gallo, E., Fender, R., \& Pooley, G. G. 2003, MNRAS, 344, 60

Gallo, E., Fender, R. P., Miller-Jones, J. C. A., et al. 2006, MNRAS, 370, 1351

Gallo, E., Miller, B., \& Fender, R. 2012, MNRAS, 423, 590

Gallo, E., Miller-Jones, J. C. A., Russell, D. M., et al. 2014, MNRAS, 445, 290

Gehrels, N. 1986, ApJ, 303, 336

Hannikainen, D. C., Charles, P. A., van Zyl, L., et al. 2005, MNRAS, 357, 325

Hannikainen, D. C., Hunstead, R. W., Campbell-Wilson, D., \& Sood, R. K. 1998, A\&A, 337, 460

Harris, W. 1996, AJ, 112, 1487

Heinke, C., Cohn, H., \& Lugger, P. 2009, ApJ, 692, 584

Heinke, C., Sivakoff, G., Morii, M., \& Kuulkers, E. 2011, ATel, 3363, 1

Heinke, C. O., Grindlay, J. E., Edmonds, P. D., et al. 2005, ApJ, 625, 796

Helfand, D., White, R., \& Becker, R. 2015, ApJ, 801, 26

Hill, A. B., Szostek, A., Corbel, S., et al. 2011, MNRAS, 415, 235

Irrgang, A., Wilcox, B., Tucker, E., \& Schiefelbein, L. 2013, A\&A, 549, 137

Ivanova, N., Justham, S., Chen, X., et al. 2013, A\&ARv, 21, 59

Janka, H. 2013, MNRAS, 434, 1355

Jonker, P. G., \& Nelemans, G. 2004, MNRAS, 354, 355

Juric, M., Ivezic, Z., Brooks, A., et al. 2008, ApJ, 673, 864

Kalogera, V. 1999, ApJ, 521, 723

Kalogera, V., \& Webbink, R. 1998, ApJ, 493, 351

Kiel, P., \& Hurley, J. 2006, MNRAS, 369, 1152

King, A. R. 2000, MNRAS, 315, 33

King, A. R., \& Ritter, H. 1998, MNRAS, 293, 42

Kirsten, F., Vlemmings, W., Freire, P., et al. 2014, A\&A, 565, 43

Knapp, G., Gunn, G., Bowers, P., \& Vasquez Poritz, J. 1996, ApJ, 462, 231

Knevitt, G., Wynn, G. A., Vaughan, S., \& Watson, M. G. 2014, MNRAS, 437, 3087

Kording, E., Rupen, M., Knigge, C., et al. 2008, Sci, 320, 1318

Kording, E. G., Knigge, C., Tzioumis, T., \& Fender, R. 2011, MNRAS, 418, 129

Kuulkers, E., Kouveliotou, C., Belloni, T., et al. 2013, A\&A, 552, 32

Liu, S., Pen, U.-L., Macquart, J., Brisken, W., \& Deller, A. 2016, arXiv:1507. 00884

Lyne, A. G., \& Manchester, R. N. 1988, MNRAS, 234, 477

Maccarone, T., Torres, M. P., Britt, C., et al. 2012, MNRAS, 426, 3057

Maccarone, T. J. 2005, MNRAS, 360, 30

Maccarone, T. J., \& Knigge, C. 2007, A\&G, 48, 12

Maccarone, T. J., \& Patruno, A. 2013, MNRAS, 428, 1335

Maccarone, T. J., Wijnands, R. A. M., Degenaar, N., et al. 2015, arXiv:1505. 02769

Machin, G., Lehto, H. J., McHardy, I. M., Callanan, P. J., \& Charles, P. A. 1990, MNRAS, 246, 237

Manchester, R. N., Hobbs, G. B., Teoh, A., \& Hobbs, M. 2005, yCat, 7245, 0

Mason, P., \& Gray, C. 2007, ApJ, 660, 662

Mathieu, R., van den Berg, M., Torres, G., et al. 2003, AJ, 125, 246

McClintock, J., \& Remillard, R. 2006, in Compact Stellar X-Ray Sources, ed. W. Lewin, \& M. van der Klis (Cambridge: Cambridge Univ. Press), 157

McMullin, J., Waters, B., Schiebel, D., Young, W., \& Golap, K. 2007, in ASP Conf. Ser. 376, Astronomical Data Analysis Software and Systems XVI, ed. R. Shaw, F. Hill, \& D. Bell (San Francisco, CA: ASP), 127

Merloni, A., Heinz, S., \& di Matteo, T. 2003, MNRAS, 345, 1057

Middelberg, E., Deller, A., Morgan, J., et al. 2011, A\&A, 526, 74

Migliari, S., \& Fender, R. 2006, MNRAS, 366, 79

Migliari, S., Fender, R. P., Rupen, M., et al. 2003, MNRAS, 342, 67

Migliari, S., Miller-Jones, J. C. A., \& Russell, D. M. 2011, MNRAS, 415, 2407

Migliari, S., Tomsick, J. A., Miller-Jones, J. C. A., et al. 2010, ApJ, 710, 1117

Miller-Jones, J., Sivakoff, G., Heinke, C., et al. 2011a, ATel, 3378, 1

Miller-Jones, J. C. A., Jonker, P. G., Maccarone, T. J., Nelemans, G., \& Calvelo, D. E. 2011b, ApJ, 739, 18

Miller-Jones, J. C. A., Sivakoff, G. R., Altamirano, D., et al. 2010, ApJ, 716, 109

Miller-Jones, J. C. A., Strader, J., Heinke, C. O., et al. 2015, MNRAS, 453, 3918

Montez, R., Jr., De Marco, O., Kastner, J., \& Chu, Y. 2010, ApJ, 721, 1820 
Moore, C. B., Rutledge, R. E., Fox, D. W., et al. 2000, ApJ, 532, 1181 Morii, M., Negoro, H., Mihara, T., et al. 2011, ATel, 3356, 1

Munar-Adrover, P., Paredes, J. M., Ribo, M., et al. 2014, ApJ, 786, 11 Narayan, R., \& Yi, I. 1994, ApJ, 452, 710

Ng, C., Romani, R., Brisken, W., Chatterjee, S., \& Kramer, M. 2007, ApJ, 654,487

O’Dell, C. R., Peimbert, M., \& Kinman, T. D. 1964, ApJ, 140, 119

Olausen, S. A., \& Kaspi, V. M. 2014, ApJS, 212, 6

Papitto, A., Ferrigno, C., Bozzo, E., et al. 2013, Natur, 501, 517

Pfahl, E., Rappaport, S., \& Podsiadlowski, P. 2003, ApJ, 597, 1036

Pooley, G. 2004, ATel, 355, 1

Portegies Zwart, S., Dewi, J., \& Maccarone, T. 2004, MNRAS, 355, 413

Portegies Zwart, S., \& Verbunt, F. 1996, A\&A, 309, 179

Portegies Zwart, S., Verbunt, F., \& Ergma, E. 1997, A\&A, 321, 207

Possenti, A., Cerutti, R., Colpi, M., \& Mereghetti, S. 2002, A\&A, 387, 993

Ransom, S. M., Stairs, I. H., Archibald, A. M., et al. 2014, Natur, 505, 520

Ratti, E. M., Jonker, P. G., Miller-Jones, J. C. A., et al. 2012, MNRAS, 423, 2656

Remillard, R., \& McClintock, J. 2006, ARA\&A, 44, 49

Repetto, S., Davies, M., \& Sigurdsson, S. 2012, MNRAS, 425, 2799

Repetto, S., \& Nelemans, G. 2015, MNRAS, 453, 3341

Romani, R. 1992, ApJ, 399, 621

Romani, R. 1994, in Neutron Stars and Black Holes in Low Mass Systems, Vol. 56 ed. A. Shafter (San Francisco, CA: ASP), 196

Romani, R. 1998, A\&A, 333, 583

Rupen, M., Dahwan, V., \& Mioduszewski, A. 2005, ATel, 524, 1

Rupen, M., Dhawan, V., \& Mioduszewski, A. J. 2002, IAUC, 7893, 2
Russell, D. M., Markoff, S., Casella, P., et al. 2013a, MNRAS, 429, 815

Russell, T. D., Miller-Jones, J. C. A., Sivakoff, G. R., et al. 2016, arXiv:1605. 07136

Russell, D. M., Russell, T. D., Miller-Jones, J. C. A., et al. 2013b, ApJL, 768, L35

Russell, T. D., Soria, R., Motch, C., et al. 2014, MNRAS, 439, 1381

Rutledge, R., Moore, C., Fox, D., \& Lewin, W. 1998, ATel, 8, 1

Sarajedini, A., Bedin, L., Chaboyer, B., et al. 2007, AJ, 133, 1658

Seaquist, E. 1993, RPPh, 56, 1145

Shahbaz, T., Charles, P., \& King, A. 1998, MNRAS, 301, 382

Shakura, N., \& Sunyaev, R. A. 1973, A\&A, 24, 337

Skrutskie, M. F., Cutri, R. M., Stiening, R., et al. 2006, AJ, 131, 1163

Strader, J., Chomiuk, L., Maccarone, T., et al. 2012a, ApJ, 750, 27

Strader, J., Chomiuk, L., Maccarone, T. J., Miller-Jones, J. C. A., \& Seth, A. 2012b, Natur, 490, 71

Tetarenko, A. J., Bahramian, A., Sivakoff, G., et al. 2016a, MNRAS, 460, 345

Tetarenko, B., Sivakoff, G., Heinke, C., \& Gladstone, J. C. 2016b, ApJS, 222, 15

Tudose, V., Fender, R. P., Linares, M., Maitra, D., \& van der Klis, M. 2009, MNRAS, 400, 2111

van den Bosch, R., de Zeeuw, T., Gebhardt, K., Noyola, E., \& van de Ven, G. 2006, ApJ, 641, 852

White, N., \& Angelini, L. 2001, ApJ, 561, 101

White, N., \& Holt, S. 1982, ApJ, 257, 318

Wu, Y. X., Yu, W., Li, T. P., Maccarone, T. J., \& Li, X. D. 2010, ApJ, 718,620

Yungelson, L. R., Lasota, J., Nelemans, G., et al. 2006, A\&A, 454, 559 